\title{
Intermittency in two-dimensional Ekman-Navier-Stokes turbulence
}

\author{
G. Boffetta, ${ }^{1,2}$ A. Celani, ${ }^{3}$ S. Musacchio, ${ }^{1,2}$ and M. Vergassola ${ }^{4}$ \\ ${ }^{1}$ Dipartimento di Fisica Generale, Università di Torino, Via Pietro Giuria 1, 10125 Torino, Italy \\ ${ }^{2}$ INFM Sezione di Torino Università, Corso Raffaello 30, 10125 Torino, Italy \\ ${ }^{3}$ CNRS, INLN, 1361 Route des Lucioles, 06560 Valbonne, France \\ ${ }^{4}$ CNRS, Observatoire de la Côte d'Azur, Boîte Postale 4229, 06304 Nice Cedex 4, France
}

(Received 29 November 2001; revised manuscript received 7 February 2002; published 16 August 2002)

\begin{abstract}
We study the statistics of the vorticity field in two-dimensional Navier-Stokes turbulence with linear Ekman friction. We show that the small-scale vorticity fluctuations are intermittent, as conjectured by Bernard [Europhys. Lett. 50, 333 (2000)] and Nam et al. [Phys. Rev. Lett. 84, 5134 (2000)]. The small-scale statistics of vorticity fluctuations coincide with that of a passive scalar with finite lifetime transported by the velocity field itself.
\end{abstract}

In many physical situations, the incompressible flow of a shallow layer of fluid can be described by the twodimensional Navier-Stokes equations supplemented by a linear damping term which accounts for friction. An important instance, among others, relevant to geophysical applications is the rotating flow subject to Ekman friction [1]. The twodimensional Ekman-Navier-Stokes equation are written in terms of a single scalar field, the vorticity $\omega=\boldsymbol{\nabla} \times \boldsymbol{v}$,

$$
\frac{\partial \omega}{\partial t}+\boldsymbol{v} \cdot \nabla \omega=\nu \nabla^{2} \omega-\alpha \omega+f_{\omega},
$$

where $\nu$ is the fluid viscosity and $\alpha$ is the Ekman friction coefficient. In two dimensions, the incompressible velocity field $\boldsymbol{v}$ can be expressed in terms of the stream function $\psi$ as $\boldsymbol{v}=\left(\partial_{y} \psi,-\partial_{x} \psi\right)$. The vorticity and stream function are therefore related by $\omega=-\Delta \psi$. The term $f_{\omega}$ is an external source of energy acting on the largest scales-e.g., stirring. This term counteracts the dissipation by viscosity and friction and allows one to obtain a statistically steady state, characterized by a total enstrophy $Z=\left\langle\omega^{2}\right\rangle$.

Here, we will study the statistical properties of vorticity fluctuations $\delta_{r} \omega=\omega(\boldsymbol{x}+\boldsymbol{r}, t)-\omega(\boldsymbol{x}, t)$ at scales $r$ smaller than the correlation length $L$ of the external forcing. We will show that of the statistics of $\delta_{r} \omega$ is intermittent, and that the vorticity field has the same scaling properties as a passive scalar with a finite lifetime.

As shown in Fig. 1, the vorticity field-resulting from the numerical integration of Eq. (1), see Ref. [2] for details-is characterized by filamental structures whose thickness can be as small as the smallest active length scales. The wide range of scales involved in the vorticity dynamics manifests itself in the appearance of power-law scaling for the spectrum of vorticity fluctuations $Z(k)=2 \pi k\left\langle|\hat{\omega}(\boldsymbol{k})|^{2}\right\rangle \sim k^{-1-\xi}$. As already shown by Nam et al. [4], the spectral slope $-1-\xi$ depends on the intensity of the Ekman drag: for the frictionless Navier-Stokes case $(\alpha=0)$ we have $\xi=0$; a nonvanishing friction regularizes the flow depleting the formation of small-size structures and results in a steeper spectrum (see Fig. 2).

In the range $0<\xi<2$ the exponent $\xi$ coincides with the scaling exponent $\zeta_{2}$ of the second-order moment of vorticity fluctuations, $S_{2}^{\omega}(r)=\left\langle\left(\delta_{r} \omega\right)^{2}\right\rangle \sim r^{\zeta_{2}}$. Let us now focus on a given value of $\alpha$. In Fig. 3 we show the probability density functions of vorticity fluctuations $\delta_{r} \omega$ at various $r$, rescaled by their rms value $\left\langle\left(\delta_{r} \omega\right)^{2}\right\rangle^{1 / 2}$. As the separation decreases, we observe that the probability of observing very weak or very intense vorticity excursions increases at the expense of fluctuations of average intensity. This phenomenon goes under the name of intermittency. Its visual counterpart is the organization of the field into "quiescent" areas (the patches, where the vorticity changes smoothly) and "active" regions (the filaments, across which the vorticity experiences relatively strong excursions).

The dynamical origin of this phenomenon can be understood as follows (see also Refs. [3,4]). Let us first notice that, for any $\alpha$ strictly positive and as far as the statistical properties in the scaling range are concerned, we can disregard the viscous term in Eq. (1). Indeed, at variance with the frictionless case, where the enstrophy flux is constant in the scaling range, in the presence of friction it decays as $k^{-\xi}$ (see Fig. 4). At the viscous wave number $k_{d} \sim \nu^{-1}$ the enstrophy flux is stopped by viscous dissipation, with a viscous dissipation rate $\bar{\epsilon}_{\omega} \sim \nu^{\xi}$. Therefore, the enstrophy dissipation vanishes in the inviscid limit $\nu \rightarrow 0$, since $\xi>0$ (see Fig. 2).

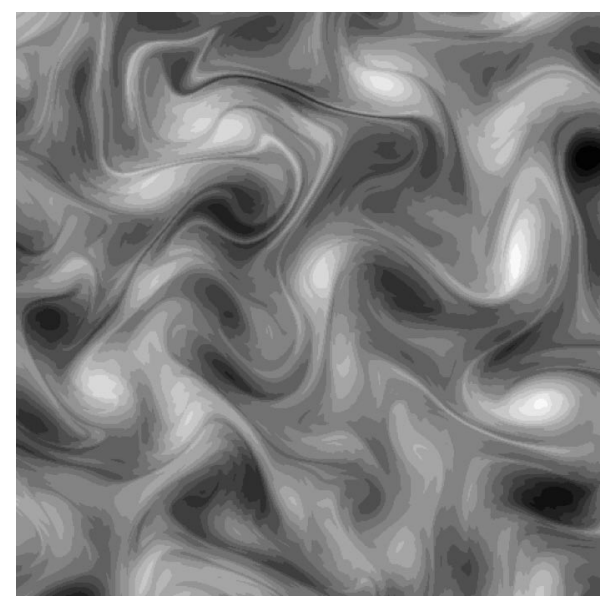

FIG. 1. Snapshot of the vorticity field resulting from the numerical integration of Eq. (1). Details are given in Ref. [2]. 


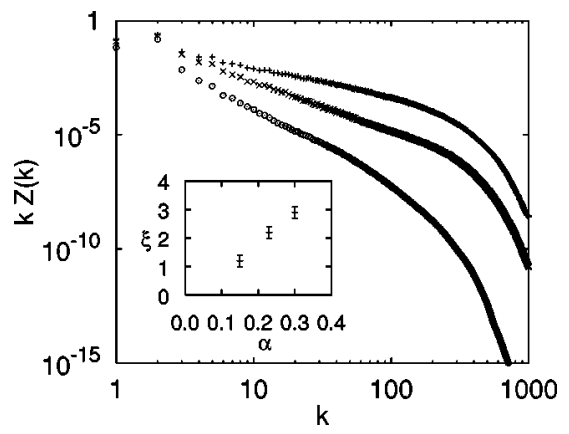

FIG. 2. The vorticity spectrum $Z(k) \sim k^{-1-\xi}$ becomes steeper by increasing the Ekman coefficient $\alpha$. Here $\alpha=0.15(+), \alpha$ $=0.23(\times)$, and $\alpha=0.30(\odot)$. In the inset, the exponent $\xi$ is shown as a function of $\alpha$.

In other words, in the limit of vanishingly small viscosity, there is no dissipative anomaly [3], and Eq. (1) can be solved by the method of characteristics yielding the expression $\omega(\boldsymbol{x}, t)=\int_{-\infty}^{t} f_{\omega}(\boldsymbol{X}(s), s) \exp [-\alpha(t-s)] d s$, where $\boldsymbol{X}(s)$ denotes the trajectory of a particle transported by the flow, $\dot{\boldsymbol{X}}(s)=\boldsymbol{v}(\boldsymbol{X}(s), s)$, ending at $\boldsymbol{X}(t)=\boldsymbol{x}$. The uniqueness of the trajectory $\boldsymbol{X}(s)$ in the limit $\nu \rightarrow 0$ is ensured by the fact that the velocity field is Lipschitz continuous, as it can be seen from the velocity spectrum $E(k)=Z(k) / k^{2} \sim k^{-3-\xi}$, always steeper than $k^{-3}$ (see Fig. 2). We remark that for $\xi>0$ the second-order velocity structure function is dominated by the IR contribution of the spectrum and thus trivially displays smooth scaling independently of the value of $\xi$. This is not the case for odd order structure functions that, in the absence of enstrophy dissipative anomaly, display anomalous scaling at the leading order [5]. We have checked that this is indeed the case in our simulations (not shown).

Vorticity differences are then associated with couples of particles $\omega\left(\boldsymbol{x}^{\prime}, t\right)-\omega(\boldsymbol{x}, t)=\int_{-\infty}^{t}\left[f_{\omega}\left(\boldsymbol{X}^{\prime}(s), s\right)\right.$ $\left.-f_{\omega}(\boldsymbol{X}(s), s)\right] \exp [-\alpha(t-s)] d s$. Inside the time integral, the difference between the value of $f_{\omega}$ at $\boldsymbol{X}^{\prime}$ and that at $\boldsymbol{X}$ is negligibly small as long as the two particles lie at a distance smaller than $L$, the correlation length of the forcing; conversely, when the pair is at a distance larger than $L$, it approximates a Gaussian random variable $\Omega$. We then have $\delta_{r} \omega \sim \Omega \int_{-\infty}^{t-T_{L}(r)} \exp [-\alpha(t-s)] d s \sim \Omega \exp \left[-\alpha T_{L}(r)\right]$, where $T_{L}(r)$ is the time that a couple of particles at distance $r$ at

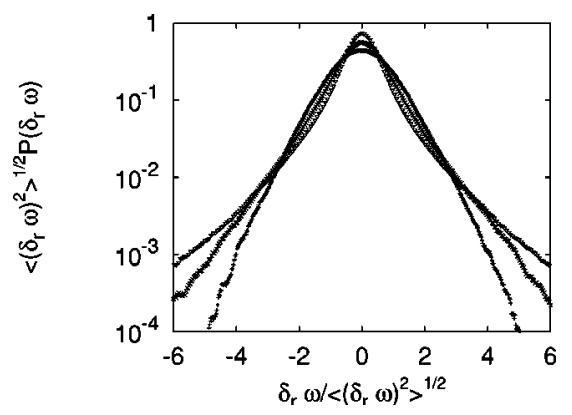

FIG. 3. Probability density functions of normalized vorticity increments $\delta_{r} \omega /\left\langle\left(\delta_{r} \omega\right)^{2}\right\rangle^{1 / 2}$. Here, $r=0.20(+), r=0.07(\times), r$ $=0.02(\nabla)$. For large separations the statistics is close to Gaussian, becoming increasingly intermittent for smaller $r$.

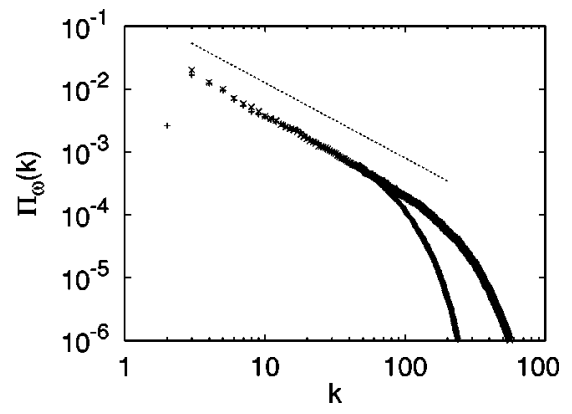

FIG. 4. Enstrophy flux $\Pi_{\omega}(k) \sim k^{-\xi}$ for $\nu=5 \times 10^{-5}(+)$ and $\nu=1.5 \times 10^{-5}(\times)$. Here $\alpha=0.15$. Reducing $\nu$, the remnant enstrophy flux at small scales tends to zero as $\nu^{\xi}$ (see text), allowing to disregard viscous dissipation.

time $t$ take to reach a separation $L$ (backward in time). Large vorticity fluctuations arise from couples of particles with relatively short exit times $T_{L}(r) \ll\left\langle T_{L}(r)\right\rangle$, whereas small vorticity fluctuations are associated to large exit times.

Since the velocity field is smooth, two dimensional, and incompressible, particles separate exponentially fast and their statistics can be described in terms of the finite-time Lyapunov exponent $\gamma$. For large times, the random variable $\gamma$ reaches a distribution $P(\gamma, t) \sim t^{1 / 2} \exp [-G(\gamma) t]$. The Cramér function $G(\gamma)$ is concave, positive, with a quadratic minimum in $\lambda$ (the maximum Lyapunov exponent), $G(\lambda)$ $=0$, and its shape far from the minimum depends on the details of the velocity statistics [6-8]. The finite-time Lyapunov exponent and exit times are related by the condition $L=r \exp \left[\gamma T_{L}(r)\right]$. That allows to obtain for $r \ll L$ the following estimate for moments of vorticity fluctuations

$$
S_{p}^{\omega}(r) \sim\left\langle\Omega^{p}\right\rangle \int d \gamma\left(\frac{r}{L}\right)^{[p \alpha+G(\gamma)] / \gamma} \sim\left(\frac{r}{L}\right)^{\zeta_{p}^{\omega}} .
$$

The scaling exponents are evaluated from Eq. (2) by a steepest descent argument as $\zeta_{p}^{\omega}=\min _{\gamma}\{p,[p \alpha+G(\gamma)] / \gamma\}$. Intermittency manifests itself in the nonlinear dependence of the exponents $\zeta_{p}^{\omega}$ on the order $p$. It has to be noticed that the active

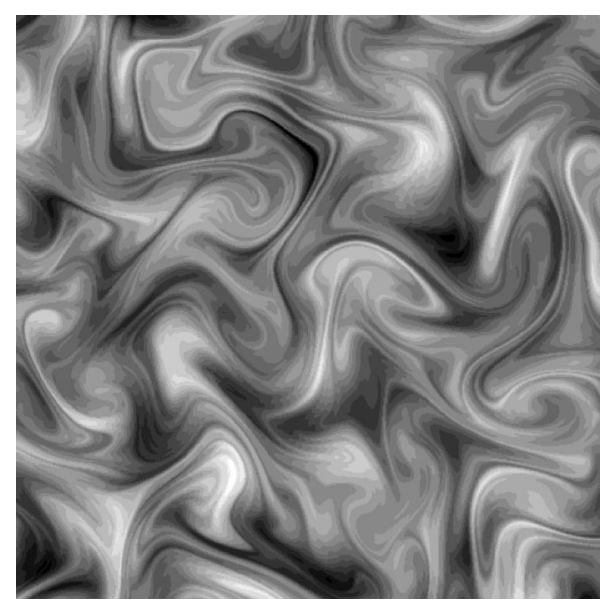

FIG. 5. Snapshot of the passive scalar field, simultaneous to the vorticity field shown in Fig. 1 


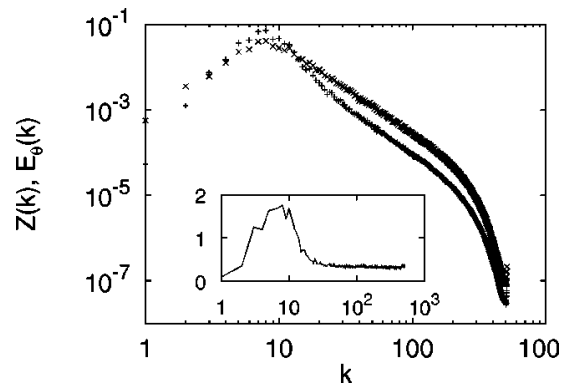

FIG. 6. Power spectra of passive scalar $(X)$ and vorticity $(+)$. Here $\alpha=0.15$. In the inset we show the ratio $Z(k) / E_{\theta}(k)$, which approaches a constant for large $k$.

nature of $\omega$ has been completely ignored in the above arguments: the crucial hypothesis in the derivation of Eq. (2) is that the statistics of trajectories be independent of the forcing $f_{\omega}$. This is quite a nontrivial assumption, since it is clear that forcing may affect large-scale vorticity and thus influence velocity statistics, but it can be justified by the following argument. The random variable $\Omega$ arises from forcing contributions along the trajectories at times $s<t-T_{L}(r)$, whereas the exit time $T_{L}$ is clearly determined by the evolution of the strain at times $t-T_{L}(r)<s<t$. Since the correlation time of the strain is $\alpha^{-1}$, for $T_{L}(r) \gg 1 / \alpha$ we might expect that $\Omega$ and $T_{L}(r)$ be statistically independent. This condition can be translated in terms of the finite-time Lyapunov exponent as $r \ll L \exp (-\gamma / \alpha)$ and thus at sufficiently small scales it is reasonable to consider $\omega$ as a passive field. We remark that, if the velocity field was nonsmooth, the exit times would be independent of $r$ in the limit $r \rightarrow 0$ and the above argument would not be relevant. Therefore, the smoothness of the velocity field plays a central role in the equivalence of vorticity and passive scalar statistics for this system.
To directly check whether small-scale vorticity can be considered as passively advected by velocity, we also solved the equation of transport of the passive scalar (Fig. 5) with a finite lifetime [3,9-11],

$$
\frac{\partial \theta}{\partial t}+\boldsymbol{v} \cdot \nabla \theta=\nu \nabla^{2} \theta-\alpha \theta+f_{\theta},
$$

where the velocity field results from the parallel integration of Eq. (1). The parameters appearing in Eqs. (1) and (3) are the same, yet the forcings $f_{\omega}$ and $f_{\theta}$ are independent processes with the same statistics. According to the picture drawn above, we expect to observe the same small-scale statistics for $\delta_{r} \omega$ and $\delta_{r} \theta=\theta(\boldsymbol{x}+\boldsymbol{r}, t)-\theta(\boldsymbol{x}, t)$.

In Fig. 6 we show the power spectra of vorticity $Z(k)$ and of the passive scalar $E_{\theta}(k)$. The estimate of the range of wave numbers at which the statistics of vorticity and passive scalar are expected to be coincident is $k \gg k^{*} \simeq k_{f} \exp (\lambda / \alpha)$. With the actual values $k_{f}=8, \alpha=0.15$, and $\lambda=0.16$ (see inset of Fig. 8) we have $k^{*} \simeq 23$. The two curves in Fig. 6 are indeed parallel at large $k\left(k \gg k^{*}\right)$, in agreement with the expectation $\zeta_{2}^{\omega}=\zeta_{2}^{\theta}$. At smaller wave numbers we observe a big bump in $Z(k)$ around $k=k_{f}$, which has no correspondent in $E_{\theta}(k)$. This deviation is most likely associated to the presence of an inverse energy flux in the Navier-Stokes equation, a phenomenon that has no equivalent in the passive scalar case. Due to this effect, the scaling quality of $S_{p}^{\omega}(r)$ is poorer than the $S_{p}^{\theta}(r)$ one, and a direct comparison of scaling exponents in physical space is even more difficult. However, we observe in Fig. 7 that the probability density functions of vorticity and passive scalar increments, once rescaled by their root-mean-square fluctuation, collapse remarkably well
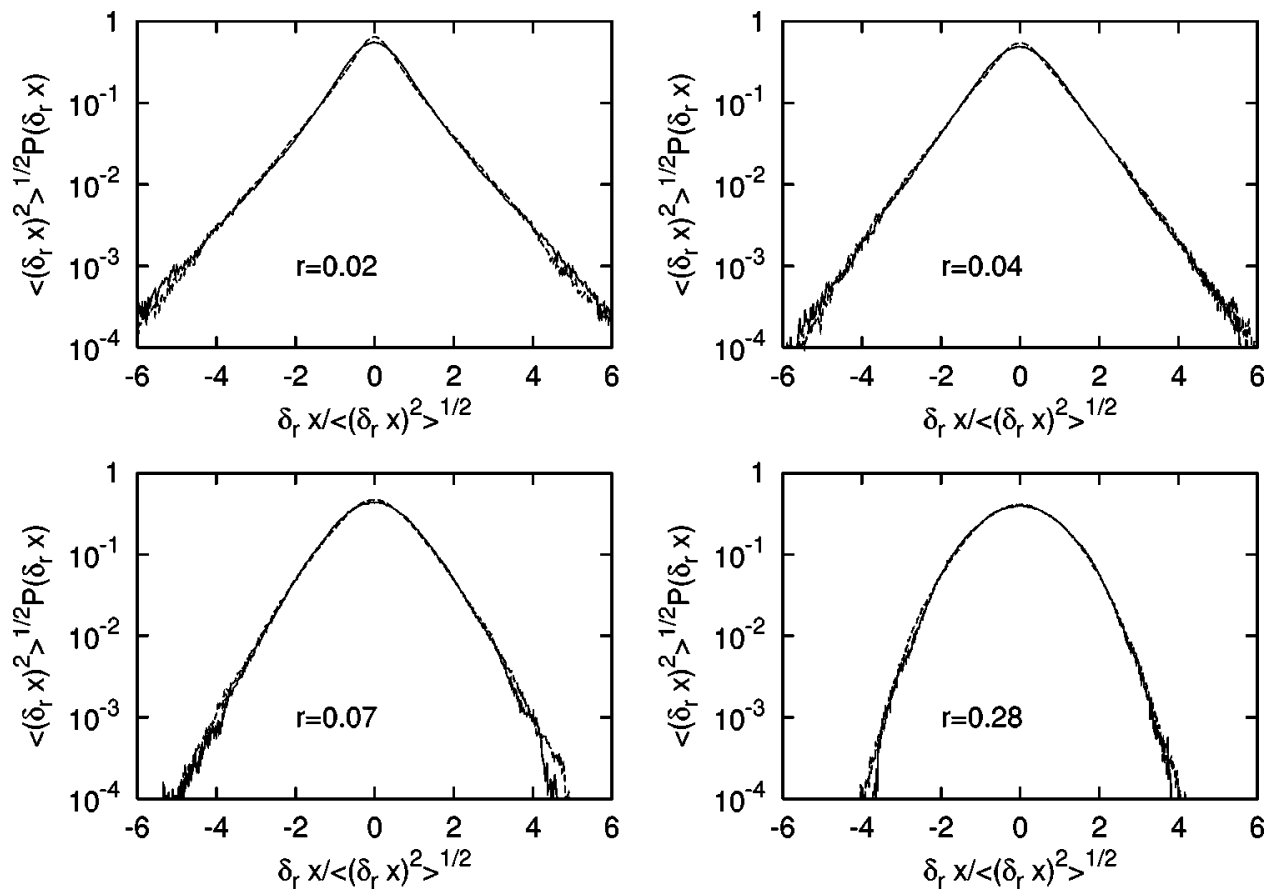

FIG. 7. Probability density functions of vorticity differences (solid line) and of passive scalar ones (dashed line), normalized by their respective standard deviation at different scales $r$ within the scaling range. 


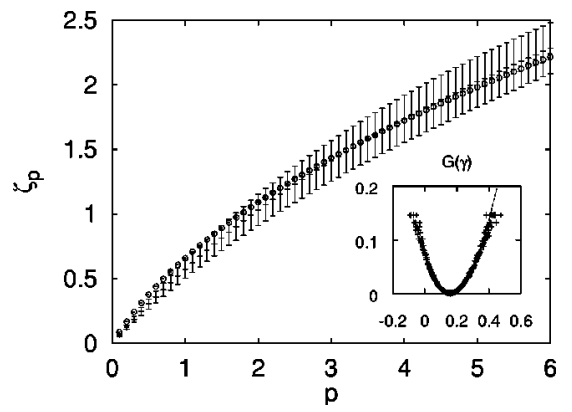

FIG. 8. The scaling exponents of the passive scalar $\zeta_{p}^{\theta}(+)$. We also show the exponents obtained from the exit-times statistics $(\odot)$ according to $\left\langle\exp \left[-\alpha p T_{L}(r)\right]\right\rangle \sim r^{\zeta^{\theta}}$, with an average over about $2 \times 10^{5}$ couples of Lagrangian particles. The error bars are estimated by the rms fluctuation of the local slope. In the inset we plot the Cramer function $G(\gamma)$ computed from finite-time Lyapunov exponents (symbols) and exit-time statistics (line).

onto each other. That is sufficient to state, along with the result $\zeta_{2}^{\omega}=\zeta_{2}^{\theta}$ obtained from Fig. 6 , the equality of the scaling exponents of the vorticity and passive scalar at any order: $\zeta_{p}^{\omega}=\zeta_{p}^{\theta}$. The actual values can be directly extracted from the statistics of the passive scalar, which is not spoiled by largescale objects. In Fig. 8 we plot the exponents $\zeta_{p}^{\theta}$ as obtained by looking at the local slopes of the structure functions $S_{p}^{\theta}(r)$. The numerical values for $\zeta_{p}^{\theta}$ are validated by the almost perfect agreement with the Lagrangian exit-time statistics.

In conclusion, we have shown that in the two-dimensional Ekman-Navier-Stokes turbulence, the small-scale vorticity fluctuations are intermittent. Intermittency is the consequence of the competition between the exponential separation of Lagrangian trajectories and the exponential decay of fluctuations due to friction. Small-scale vorticity fluctuations behave statistically as a passive scalar, as it has been confirmed by a direct comparison. The smoothness of the velocity field appears to be a crucial ingredient for the equality of active and passive scalar statistics.

This work was supported by the EU under Contract Nos. HPRN-CT-2000-00162 and FMRX-CT-98-0175, and by the MIUR-Cofin2001, Contract No. 2001023848. Numerical simulations were performed at IDRIS (Project Nos. 011226 and 011411) and at CINECA (INFM Progetto Calcolo Parallelo).
[1] R. Salmon, Geophysical Fluid Dynamics (Oxford University Press, New York, 1998); other well known examples are the Rayleigh friction in stratified fluids, the Hartmann friction in magneto-hydrodynamics [J. Sommeria, J. Fluid Mech. 170, 139 (1986)], and the friction induced by surrounding air in soap films [M. Rivera and X.L. Wu, Phys. Rev. Lett. 85, 976 (2000)].

[2] The numerical integration of Eq. (1) starting from a zero field is performed by a fully dealiased pseudospectral code with a second-order Runge-Kutta scheme, on a doubly periodic square domain of size $L=2 \pi$ at different resolutions: $N^{2}$ $=512^{2}, 1024^{2}, 2048^{2}$ grid points. A small viscosity (depending on the resolution: $\nu=5 \times 10^{-5}$ for $N=512, \nu=1.5 \times 10^{-5}$ for $N=1024$, and $\nu=5 \times 10^{-6}$ for $N=2048$ ) is used to remove the remnant enstrophy flux at small scales. The large-scale forcing $f_{\omega}$ is Gaussian, $\delta$ correlated in time, and limited to a shell of wave numbers around $k_{f}=2 \pi / L \quad\left(k_{f}=2\right.$ for $N$ $=512,2048$, and $k_{f}=8$ for $\left.N=1024\right)$. Forcing amplitude is chosen to provide an enstrophy injection rate $F=0.16$. At vari- ance with other choices for $f_{\omega}$ commonly used (e.g., largescale shear), this kind of forcing ensures the statistical isotropy and homogeneity of the vorticity field.

[3] K. Nam, E. Ott, T.M. Antonsen, and P.N. Guzdar, Phys. Rev. Lett. 84, 5134 (2000).

[4] M. Chertkov, Phys. Fluids 10, 3017 (1998).

[5] D. Bernard, Europhys. Lett. 50, 333 (2000).

[6] E. Ott, Chaos in Dynamical Systems (Cambridge University Press, Cambridge, England, 1993).

[7] T. Bohr, M. H. Jensen, G. Paladin, and A. Vulpiani, Dynamical Systems Approach to Turbulence (Cambridge University Press, Cambridge, England, 1998).

[8] M. Chertkov, Phys. Fluids 11, 2257 (1999).

[9] S. Corrsin, J. Fluid Mech. 11, 407 (1961).

[10] K. Nam, T.M. Antonsen, P.N. Guzdar, and E. Ott, Phys. Rev. Lett. 83, 3426 (1999).

[11] Z. Neufeld, C. Lopez, E. Hernandez-Garcia, and T. Tel, Phys. Rev. E 61, 3857 (2000). 